\title{
Caracterización genotípica por MLST de Ornithobacterium rhinotracheale procedentes de aves de corral en el Perú
}

\author{
Genotypic characterization by MLST of Ornithobacterium rhinotracheale \\ from poultry in Peru
}

\author{
Rony Yunior Cotaquispe Nalvarte, 1,5 Alberto Manchego Sayan ${ }^{2}$, \\ Jorge Rodríguez Bailon ${ }^{3}$, Ysabel Koga Yanagui ${ }^{4}$
}

\section{Resumen}

El objetivo del presente estudio fue la genotipificación de Ornithobacterium rhinotracheale. Se identificaron 36 cepas por pruebas moleculares y se realizó la genotipificación por Multilocus Sequence Typing (MLST). Los ensayos identificaron las secuencias tipo: N.T.1, N.T2 y N.T3, diferentes a la base de datos del NCBI y 19 alelos distintos con 229 sitios polimórficos. Además, 32 cepas pertenecen al genotipo común ST1. Los hallazgos identificaron al menos cuatro genotipos en pollos de varias regiones del Perú.

Palabras clave: genotipo, secuencia tipo, Multilocus Sequence Typing

${ }^{1}$ Laboratorio de Biología Molecular Bioservice SRL, Lima, Perú

${ }^{2}$ Laboratorio de Inmunología Molecular, Facultad de Medicina Veterinaria, Universidad Nacional Mayor de San Marcos, Lima, Perú

${ }^{3}$ Universidad Peruana Cayetano Heredia, Lima, Perú

${ }^{4}$ Departamento de Investigación, Bioservice SRL, Lima, Perú

${ }^{5}$ E-mail: r.cotaquispe.n@gmail.com

Recibido: 24 de noviembre de 2020

Aceptado para publicación: 15 de mayo de 2021

Publicado: 24 de agosto de 2021

CLos autores. Este artículo es publicado por la Rev Inv Vet Perú de la Facultad de Medicina Veterinaria, Universidad Nacional Mayor de San Marcos. Este es un artículo de acceso abierto, distribuido bajo los términos de la licencia Creative Commons Atribución 4.0 Internacional (CC BY 4.0) [https:// creativecommons.org/licenses/by/4.0/deed.es] que permite el uso, distribución y reproducción en cualquier medio, siempre que la obra original sea debidamente citada de su fuente original 
The aim of this study was the genotyping of Ornithobacterium rhinotracheale. For this, 36 strains were identified by molecular testing and genotyping was performed by Multilocus Sequence Typing (MLST). The tests identified the type sequences: N.T.1, N.T2 and N.T3, different from the NCBI database and 19 different alleles with 229 polymorphic sites. In addition, 32 strains belonged to the common ST1 genotype. The findings identified at least four genotypes in chickens from various regions of Peru.

Key words: genotype, type sequence, Multilocus Sequence Typing

\section{INTRODUCCIÓN}

Ornithobacterium rhinotracheale (ORT) es un patógeno aviar presente en el tracto respiratorio de diversas especies de aves domésticas y silvestres (Vandamme et al., 1994; Hafez y Reinhard, 1999). La infección puede causar una alta mortalidad y pérdidas económicas para los avicultores (Vandamme et al., 1994; Macagnan, 2006).

$O$. rhinotracheale pertenece al orden Flavobacteriales, familia Flavobacteriaceae y género Ornithobacterium, que tiene una única especie. O. rhinotracheale (Vandamme et al., 1994; Van Empel y Hafez, 1999) y fue aislada por primera vez en 1983 por Vandamme et al. (1994). Se describió como un bacilo gramnegativo pleomórfico, de colonias convexas, lisas, hemolíticas (Van Empel y Hafez, 1999), habiéndose reportado en numerosos países (Amonsin et al., 1997; Sakai et al., 2000; Hung y Alvarado, 2001; Van Veen et al., 2001; Turan y Ak, 2002; Koga y Zavaleta, 2005; Hassanzadeh et al., 2010; Espinosa et al., 2011). Actualmente se conocen 18 serotipos nombradas con letras de la A hasta la R, y son determinadas por métodos serológicos (Van Empel et al., 1997; Turan y Ak, 2002). Algunos serovares (A, B, $D, E)$ causan reacciones cruzadas a nivel serológico, indicando la posibilidad de que existan subespecies de esta bacteria (Macagnan, 2006; Hafez y Vandamme, 2011).
El uso del PCR para la detección molecular de $O$. rhinotracheale en el Perú ha mostrado buenos resultados (Hung y Alvarado, 2001), y además permite detectar las variaciones genéticas existentes por interacciones entre serotipos peruanos mediante análisis de ADN entre los aislados de O. rhinotracheale (Koga y Zavaleta, 2005; Macagnan, 2006). De otra parte, métodos de tipificación molecular como el Multilocus Sequence Typing - MLST están disponibles y son los métodos de elección para identificación de los genotipos existentes (Sakai et al., 2000).

La tipificación molecular basada en secuencias multilocus (MLST) permite caracterizar aislados de especies bacterianas utilizando secuencias conservadas de siete genes housekeeping: ( $a d k)$ : Adenylato kinasa, (aroE): Shikimate 5-dehydrogenase, (fumC): Fumarase class II, $(g d h A)$ : Glutamate dehydrogenase, (pgi): Glucose-6-phosphate isomerase, ( $\mathrm{pmi})$ : Phosphomannose isomerase (Coll et al., 2005; Thieme et al., 2016a). Las bases de datos disponibles para secuencias MLST permiten la fácil comparación de perfiles alélicos entre aislados y/o clonas (Thieme et al., 2016a,b; Pubmlst.org, 2018).

En el Perú se carece de estudios de tipificación actuales que permitan determinar la variabilidad genética y la presencia de 
variantes genéticas en $O$. rhinotracheale. El objetivo del trabajo fue genotipificar 36 cepas de $O$. rhinotracheale procedentes de cinco regiones del Perú mediante MLST.

\section{MATERIALes y Métodos}

\section{Material Biológico}

Se utilizaron 36 cepas de $O$. rhinotracheale, aisladas de muestras clínicas de aves con signos clínicos y lesiones histopatológicas compatibles con la infección bacteriana entre 2015 y 2017, provenientes de Lima, Libertad, Arequipa, Ica y Ucayali. Las muestras correspondieron a pulmón, hígado, sacos aéreos, corazón y cerebro. Las cepas fueron aisladas de gallinas de postura (Hyline) $(\mathrm{n}=9)$, broilers $(\mathrm{n}=4)$, gallinas reproductoras $(n=22)$ y de gallos de pelea $(n=1)$.

Se realizó una impronta de cada órgano e hisopado en una placa de agar sangre y luego, con un asa de siembra se agotó la muestra por estrías en el medio de cultivo, incubándose a $37^{\circ} \mathrm{C}$ durante $24-48 \mathrm{~h}$ en jarra con cuatro velas (microaerófilo), aunque se reconoce que existen ORT que necesitan más de 48 horas de cultivo. Se consideraron como colonias sospechosas aquellas que eran puntiformes, pequeñas, grises a grises-blanquecinas, opacas, de 1-3 mm de diámetro, convexas, de superficie lisa, hemolítica o no hemolítica (Soriano et al., 2003). Las cepas con características microbiológicas idénticas a $O$. rhinotracheale fueron liofilizadas y almacenados en el banco de cepas del laboratorio de Bioservice.

\section{Extracción de ADN de O. rhinotracheale}

Se extrajo el ADN genómico de las 36 cepas utilizando el método de membranas de sílica gel con el GF-1 Tissue DNA Extraction Kit (Vivantis), de acuerdo con las indicaciones del laboratorio fabricante.

\section{Detección Molecular de $\boldsymbol{O}$. rhinotracheale}

Las cepas de $O$. rhinotracheale fueron detectadas mediante amplificación de PCR. Se utilizó un fragmento de 784 bp del gen 16S $r R N A$, utilizando los cebadores forward: OR16S - F1 (5'GAGAATTAATTTACGGATTAAG) y reverse: OR16S-R1 (5'TTCGCTTGGTCTCCGAAGAT). La reacción de PCR se realizó en un volumen final de $20 \mu \mathrm{l}$ conteniendo 10X Buffer PCR, $25 \mathrm{mM}$ de dNTPs, $25 \mathrm{mM}$ de $\mathrm{MgCl}_{2}, 10 \mathrm{pmol}$ de cada cebador, $1 \mathrm{U}$ Taq DNA polimerasa (Fermentas) y $30 \mathrm{ng}$ de ADN genómico. Se emplearon los siguientes ciclos termales: Un ciclo de desnaturalización inicial de $95{ }^{\circ} \mathrm{C}$ durante 4 min, 35 ciclos de desnaturalización a $94{ }^{\circ} \mathrm{C}$ durante $30 \mathrm{~s}$, alineamiento de $58^{\circ} \mathrm{C}$ durante $60 \mathrm{~s}, 1$ extensión de $72^{\circ} \mathrm{C}$ durante $90 \mathrm{~s}$ y 1 ciclo de extensión final de $72{ }^{\circ} \mathrm{C}$ durante 5 min, en un termociclador Veriti Thermal Cycler (Life Technologies) (Espinosa et al., 2011; Koga y Zavaleta, 2005, Soriano et al., 2003).

\section{Secuenciamiento del Gen 16S}

Se realizó la amplificación del gen $16 \mathrm{~S}$ mediante los cebadores: $27 \mathrm{~F}$ (5'AGAGTTTGATCMTGGCTC AG‘3) y 1492 R (5‘AAAGGAGGTGWTCCAR CC`3). Los dos productos de PCR fueron separados mediante electroforesis en gel de agarosa al $1.5 \%$ en buffer TBE $1 \mathrm{X}$ (Tris, Borato, EDTA) durante $45 \mathrm{~min}$ a $100 \mathrm{v}$ y visualizados bajo luz UV. Los productos se enviaron a secuenciar a la empresa Macrogen Inc. (Corea del Sur)(Lillo et al., 2006).

\section{Multilocus Sequence Typing (MLST)}

Un total de siete genes housekeeping fueron utilizados para MLST de $O$. rhinotracheale. Estos fueron seleccionados de estudios previos en aves de corral y aves silvestres por su alta semejanza con los genes de $O$. rhinotracheale (Cuadro 1). Así, dos genes (aroE y fumC) fueron seleccionados 
Cuadro 1. Genes Housekeeping, cebadores y tamaños de fragmentos usados por Multilocus Sequence Typing - MLST

\begin{tabular}{|c|c|c|c|}
\hline Gen & Síntesis & Cebador $\left(5^{\prime}-3^{\prime}\right)^{1}$ & $\begin{array}{c}\text { MLST } \\
(\mathrm{pb})\end{array}$ \\
\hline \multirow[t]{2}{*}{$a d k$} & Adenylato kinasa & F: GGCAGTGGAAAAGGAACTCA & 393 \\
\hline & & R: TCTAAACTTCCTTCGCCGTTT & \\
\hline \multirow[t]{2}{*}{$\operatorname{aroE}$} & Shikimate 5-dehydrogenase & F: GGACTCATCGGCAGAAACAT & 486 \\
\hline & & R: TGATGTTGGCATCTTGTGCT & \\
\hline \multirow[t]{2}{*}{ fumC } & Fumarase, class II & F: CACGCCACAAGGTTATGATG & 489 \\
\hline & & R: TAAACGCACGGCTTCTTCTT & \\
\hline \multirow[t]{2}{*}{$g d h A$} & Glutamate dehydrogenase/ & F: TCTGGTAGAGCACCAAACCA & 480 \\
\hline & Leucine dehydrogenase & R: GCTTGTTTTGCAACCACTCA & \\
\hline \multirow[t]{2}{*}{$m d h$} & Malate dehydrogenase & F: CGCGAAGAATTAATCGGAAC & 519 \\
\hline & (NAD) & R: CTCTTACTTGCGCAACAGCA & \\
\hline \multirow[t]{2}{*}{$p g i$} & Glucose-6-phosphate & F: AAAGCGACATTGCCAAACAT & 492 \\
\hline & isomerase & R: TTTCGAGTTCCGCTCTCACT & \\
\hline \multirow[t]{2}{*}{ pmi } & Phosphomannose isomerase & F: TGATGTGCAAGGCAATGTTT & 489 \\
\hline & & R: CTGTGTCGAGCGAAATGCTA & \\
\hline
\end{tabular}

${ }^{1} \mathrm{~F}$ - Cebador forward, R- Cebador reverse

a partir de estudios de análisis de MLEE de O. rhinotracheale (Amonsin et al., 1997) y cinco genes ( $a d k, m d h, p g i, g d h A$ y $p m i)$ a partir del análisis de MLST de Pasteurella multosida (Thieme et al., 2016a). Las secuencias de los cebadores, las condiciones de amplificación por PCR, secuencias alélicas, tipos de secuencias y aislados de referencia fueron obtenidos a partir la base de datos MLST de la Universidad de Oxford (Pubmlst.org., 2018). Los productos de PCR fueron separados por electroforesis en gel de agarosa al $1 \%$ en buffer TBE $1 \mathrm{X}$ (Tris 0.89 M, Borato 0.02 M, EDTA 0.89 M, pH 8.3) y visualizados mediante luz UV. Los productos de PCR fueron analizados en base a su tamaño de acuerdo con lo reportado en la base de datos de MLST. Los productos de PCR fueron secuenciados por ambas hebras mediante BigDye v. 3.1 sequencing terminator kit (Life Technologies) y un analizador genético ABI 3137 XL (Life Technologies). El servicio fue proveído por la empresa Macrogen Inc. (Corea del Sur) (Subaaharan et al., 2010).

\section{Curado y Alineamiento de las Secuen- cias MLST}

Las 504 secuencias de los siete genes (36 cepas) por forward y reverse se analizaron y se realizó (curado) el control de calidad de cada secuencia para garantizar los SNPs correctos y no producto de un error durante el secuenciamiento (gaps o solapamientos de nucleótidos) y eliminar los extremos cortos de secuencias que normalmente presentan errores. Se utilizó el software Chromas Lite v. 2.6.6. Luego se realizó el alineamiento de las secuencias por cada gen Housekeeping y se hizo una comparación de los sitios variables obtenidos median- 


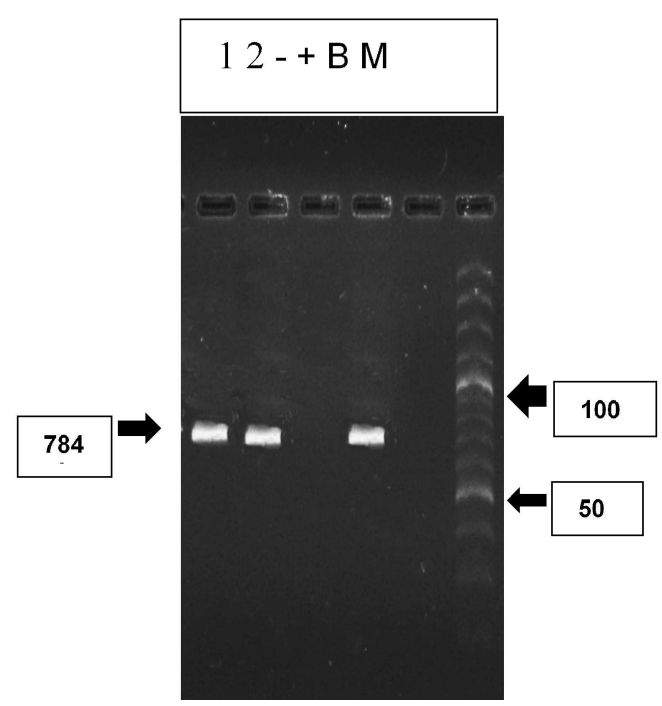

Figura 1. Detección de Ornithobacterium rhinotracheale del gen 16S rRNA. Línea 1 al 2: O. rhinotracheale, Línea 3 (control negativo): P. multocida ATCC, línea 4 (control positivo): $O$. rhinotracheale ATCC 51464, línea 5: Blanco; M, GeneRuler 100 bp Plus DNA Ladder Thermo Scientific (Thermo Scientific)

te el software MEGA v. 6.0. Las secuencias en formato FASTA se llevaron a la base de datos (Pubmlst.org., 2018).

\section{Análisis de Secuencias de MLST}

Los perfiles alélicos fueron determinados a partir de secuencias de siete genes Housekeeping de las cepas de $O$. rhinotracheale y su comparación con las secuencias tipo (ST) presentes en la base de datos MLST.

El nivel de recombinación entre secuencias fue calculado mediante el índice de asociación (IA) descrito por Maynard Smith
(Smith et al., 1993), usando el software START.2 (Jolley et al., 2001; Jolley y Maiden, 2010). Un árbol filogenético fue construido utilizando los perfiles alélicos para los siete genes concatenados, mediante el método de Neighbor Joining, disponible en el software MEGA v. 6.0 (Tamura et al., 2013; Thieme et al., 2016a,b; Pubmlst.org., 2018).

\section{Resultados}

Los resultados de las muestras secuenciadas fueron comparados con los datos de la base de datos del GenBank (Fig. 1). Los genes Housekeeping obtenidos por PCR muestran en la Figura 2.

Los 504 cromatogramas obtenidos del secuenciamiento fueron analizados mediante el software Chromas Lite a fin de discriminar y corregir las secuencias durante el curado. Posteriormente, mediante el software Mega v. 6.0 se determinaron los sitios variables y se seleccionaron alelos polimórficos, obteniéndose los sitios tipos (ST) por comparación con los perfiles alélicos almacenados en la base de datos del NCBI. Un total de cuatro ST fueron encontrados (ST1, ST NT 1, ST NT 2 y ST NT 3), siendo el perfil alélico ST1 (1, 1, 1, 1, $1,1,1)$ el más frecuente con presencia en 32 muestras de ORT, representando un $88.89 \%$ del total (Cuadro 2, Figura 3).

La diferenciación de los ST se logra mediante la similitud en los perfiles alélicos. El ST N.T1 $(1,17,7,8,7,25,1)$ difiere al perfil ST10 $(7,9,7,8,7,8,8)$ en 4 alelos. El ST N.T2 $(24,2,26,6,23,25,25)$ y N.T3 $(24$, 2, 26, 6, 2, 1, 25) son distintos al ST32 (24, 2, $26,24,23,25,25)$ en 3 y 1 alelo. Esta información determinaría tres nuevas secuencias tipo (ST) dentro de esta investigación (Cuadro 3). 


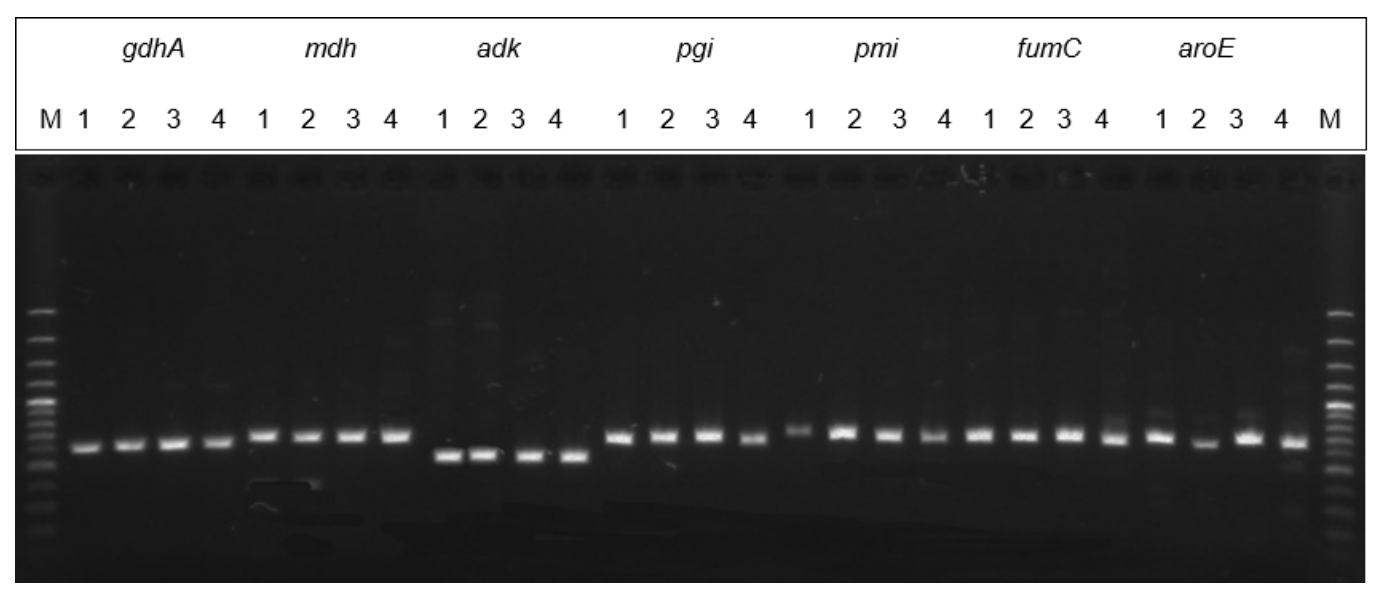

Figura 2. Los siete 7 genes Housekeeping obtenidos por PCR. Línea 1, 2, 3 y 4: Ornithobacterium rhinotracheale, (adk): Adenylato kinasa, (aroE): Shikimate 5-dehydrogenase, $(f u m C)$ : Fumarase, class II, $(g d h A)$ : Glutamate dehydrogenase, (pgi): Glucose-6-phosphate isomerase, ( $p m i)$ : Phosphomannose isomerase; $(m d h)$ Malate dehydrogenase (NAD), M, GeneRuler 100 bp Plus DNA Ladder Thermo scientific (Fermentas, EEUU)

\section{Discusión}

La variabilidad genética de $O$. rhinotracheale es mayor que en otros microorganismos (Amonsin et al., 1997; Soriano et al., 2003; Montes de Oca et al., 2018), lo cual complica la identificación eficaz de linajes clonales. En este caso, se logra una mejor caracterización molecular y agrupamiento con el método MLST (Amonsin et al., 1997; Montes de Oca et al., 2018; Tabatabai et al., 2010). Los resultados del MLST corroboran lo reportado por Thieme et al. (2016a,b), quienes identifican la prevalencia del perfil alélico ST1 en aves domésticas, en varios continentes, en tanto que Smith et al. (1993) y Thieme et al. (2016b) reportan una alta heterogeneidad de perfiles alélicos en aves silvestres. En base a estos hallazgos, este estudio apoya la probable introducción de nuevos perfiles alélicos (ST) a partir de poblaciones de aves silvestres a domésticas (Amonsin et al., 1997; Chou et al., 2009).
Los resultados del presente estudio_sugieren la presencia de moderada variabilidad genética presente en cepas de $O$. rhinotracheale provenientes de sistemas de producción avícola en el Perú, con la presencia de cuatro perfiles alélicos ST (ST1, ST N.T 1, ST N.T 2 y ST N.T 3), tres de los cuales no ha sido reportado previamente. Además, se encontró 19 alelos únicos y 229 sitios polimórficos. El menor número de sitios de polimorfismo con el estudio de Thieme et al. (2016a) se debería a la cantidad de cepas dispuestas en el trabajo. El índice de asociación (IA) calculado para las 36 cepas analizadas fue de 4.7327 (Jolley et al., 2001, 2010). Se detectó un desequilibrio de ligamiento significativo que indica estructuras de población clonal. Los resultados son comparables al estudio de Thieme et al. (2016a) donde las 36 cepas de O. rhinotracheale son clones que están estrechamente relacionadas genéticamente, pero no tienen relación con el tipo de hospedero (gallinas, pollos y gallos); por otro lado, no se podría inferir una relación geográfica debido a la cantidad de muestras analizadas. 
Cuadro 2. Perfiles alélicos de Multilocus Sequence Typing (MLST), hospedero, origen geográfico y año de aislamiento de 36 cepas de Ornithobacterium rhinotracheale

\begin{tabular}{|c|c|c|c|c|c|c|c|c|c|c|c|}
\hline \multirow[b]{2}{*}{ ق } & \multirow{2}{*}{ 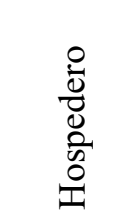 } & \multirow[b]{2}{*}{$\begin{array}{l}\overline{0} \\
.000 \\
\overrightarrow{0}\end{array}$} & \multirow{2}{*}{ 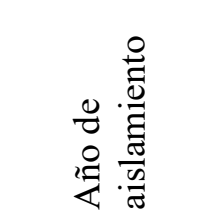 } & \multirow[b]{2}{*}{ 药 } & \multicolumn{7}{|c|}{ Perfil alélico MLST } \\
\hline & & & & & $\overrightarrow{8}$ & $\begin{array}{l}\text { lit } \\
0 \\
0\end{array}$ & 剖 & $\underset{\delta}{\mathbb{Z}}$ & $\underset{\mathbb{Z}}{\mathbb{Z}}$ & 2 & ฐ \\
\hline JRT1 & Gallo & Arequipa & LBM007-15 & 1 & 1 & 1 & 1 & 1 & 1 & 1 & 1 \\
\hline JRT2 & Gallina & Huaral & LBM 030-15 & & 1 & 1 & 1 & 1 & 1 & 1 & 1 \\
\hline JRT3 & Pollo & Huaral & LBM 053-15 & & 1 & 1 & 1 & 1 & 1 & 1 & 1 \\
\hline JRT4 & Gallina & Libertad & LBM 065-15 & & 1 & 1 & 1 & 1 & 1 & 1 & 1 \\
\hline JRT5 & Pollo & Ucayali & LBM 065-15 & & 1 & 1 & 1 & 1 & 1 & 1 & 1 \\
\hline JRT6 & Gallina & Libertad & LBM 075-15 & & 1 & 1 & 1 & 1 & 1 & 1 & 1 \\
\hline JRT7 & Pollo & Huaral & LBM 126-15 & & 1 & 1 & 1 & 1 & 1 & 1 & 1 \\
\hline JRT8 & Gallina & Lima & LBM 134-15 & & 1 & 1 & 1 & 1 & 1 & 1 & 1 \\
\hline JRT9 & Gallina & Lima & LBM 134-15 & & 1 & 1 & 1 & 1 & 1 & 1 & 1 \\
\hline IRT10 & Gallina & Lima & LBM 135-15 & & 1 & 1 & 1 & 1 & 1 & 1 & 1 \\
\hline IRT11 & Gallina & Lima & LBM $135-16$ & & 1 & 1 & 1 & 1 & 1 & 1 & 1 \\
\hline IRT12 & Gallo & Libertad & LBM 172-15 & & 1 & 1 & 1 & 1 & 1 & 1 & 1 \\
\hline IRT13 & Pollo & Libertad & LBM 172-15 & & 1 & 1 & 1 & 1 & 1 & 1 & 1 \\
\hline IRT14 & Pollo & Lima & LBM 283-15 & & 1 & 1 & 1 & 1 & 1 & 1 & 1 \\
\hline IRT15 & Pollo & Lima & LBM 283-15 & & 1 & 1 & 1 & 1 & 1 & 1 & 1 \\
\hline IRT16 & Gallina & Lima & LBM 042-16 & & 1 & 1 & 1 & 1 & 1 & 1 & 1 \\
\hline RT17 & Gallina & Arequipa & LBM 162-16 & N.T 1 & 1 & 17 & 7 & 8 & 7 & 25 & 1 \\
\hline IRT18 & Gallina & Libertad & LBM 163-16 & 1 & 1 & 1 & 1 & 1 & 1 & 1 & 1 \\
\hline IRT19 & Gallina & Lima & LBM 002-17 & & 1 & 1 & 1 & 1 & 1 & 1 & 1 \\
\hline IRT20 & Pollo & Ica & LBM 265-16 & & 1 & 1 & 1 & 1 & 1 & 1 & 1 \\
\hline IRT21 & Pollo & Huaral & LBM 032-16 & & 1 & 1 & 1 & 1 & 1 & 1 & 1 \\
\hline IRT22 & Pollo & Ica & LBM 265-16 & & 1 & 1 & 1 & 1 & 1 & 1 & 1 \\
\hline IRT23 & Pollo & Trujillo & LBM 308-16 & N.T 1 & 1 & 17 & 7 & 8 & 7 & 25 & 1 \\
\hline IRT24 & Gallina & Libertad & LBM 286-16 & 1 & 1 & 1 & 1 & 1 & 1 & 1 & 1 \\
\hline IRT25 & Gallina & Lima & LBM 168-16 & & 1 & 1 & 1 & 1 & 1 & 1 & 1 \\
\hline IRT26 & Pollo & Libertad & LBM 316-16 & & 1 & 1 & 1 & 1 & 1 & 1 & 1 \\
\hline IRT27 & Pollo & Lima & LBM 44-16 & & 1 & 1 & 1 & 1 & 1 & 1 & 1 \\
\hline IRT28 & Pollo & Ucayali & LBM 176-16 & & 1 & 1 & 1 & 1 & 1 & 1 & 1 \\
\hline IRT29 & Gallina & Libertad & LBM 286-16 & & 1 & 1 & 1 & 1 & 1 & 1 & 1 \\
\hline IRT30 & Pollo & Lima & LBM 44-16 & & 1 & 1 & 1 & 1 & 1 & 1 & 1 \\
\hline IRT31 & Pollo & Libertad & LBM 214-16 & & 1 & 1 & 1 & 1 & 1 & 1 & 1 \\
\hline IRT32 & Gallina & Libertad & LBM298-16 & & 1 & 1 & 1 & 1 & 1 & 1 & 1 \\
\hline IRT33 & Pollo & Huaral & LBM 032-16 & & 1 & 1 & 1 & 1 & 1 & 1 & 1 \\
\hline IRT34 & Gallina & Lima & LBM 249-16 & & 1 & 1 & 1 & 1 & 1 & 1 & 1 \\
\hline IRT35 & Gallina & Libertad & LBM 270-17 & N.T 2 & 24 & 2 & 26 & 6 & 23 & 25 & 25 \\
\hline RT36 & Gallina & Libertad & LBM 280-17 & N.T 3 & 24 & 2 & 26 & 6 & 2 & 1 & 25 \\
\hline
\end{tabular}

ID: identificación de la cepa; LBM: ficha de laboratorio de biología molecular 


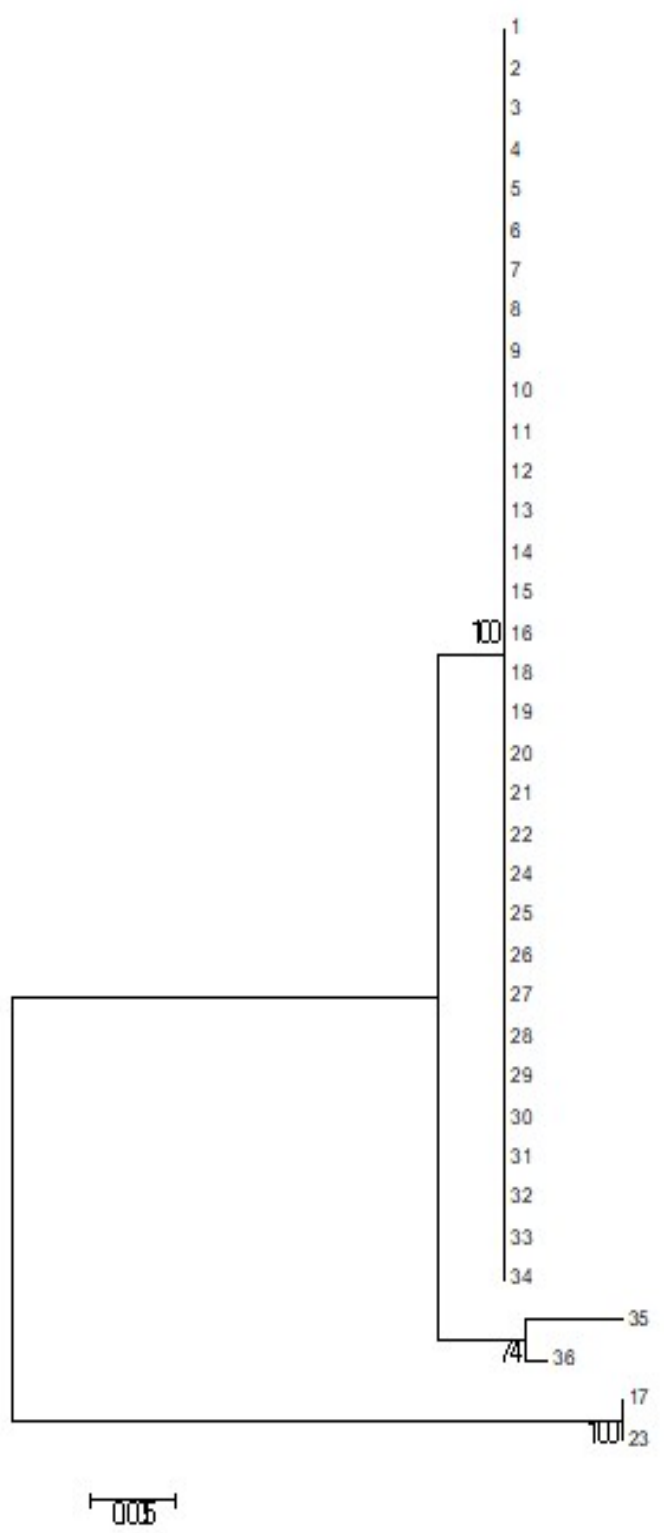

Figura 3. Árbol filogenético basado en el método estadístico (Neighbor joining) utilizando Maximum Composite Likelihood, a partir de los perfiles alélicos de MLST en 36 cepas representativas de Ornithobacterium rhinotracheale. El dendograma fue construido mediante el programa Mega 6.0

Los resultados podrían sugerir un incremento de la variabilidad genética en los últimos años con la presencia de al menos cuatro genotipos circulantes basados en el análisis de MLST. La mayor variabilidad intraespecífica observada en la región de Lima
Cuadro 3. Número de alelos y sitios variables en 229 alelos usando la base de datos pubmlst.org y Mega 6.0

\begin{tabular}{ccc}
\hline $\begin{array}{c}\text { Gene } \\
\text { Housekeeping }\end{array}$ & $\begin{array}{c}\mathrm{N}^{\circ} \text { de } \\
\text { alelos } \\
\text { diferentes }\end{array}$ & $\begin{array}{c}\mathrm{N}^{\circ} \text { de sitios } \\
\text { polimórficos }\end{array}$ \\
\hline adk & 2 & 4 \\
aroE & 3 & 14 \\
fumC & 3 & 37 \\
gdhA & 3 & 101 \\
mdh & 4 & 63 \\
pgi & 2 & 2 \\
pmi & 2 & 8 \\
\hline Total & 19 & 229 \\
\hline
\end{tabular}

(ST N.T1) guarda correspondencia con la mayor concentración de crianzas avícolas presentes en esta región; sugiriendo que la interacción entre tipos de crianza (carne / huevo) podría explicar la existencia de genotipos distintos, tal como es mencionado por otros autores (Van Empel et al., 1998; Turan y Ak, 2002; Macagnan, 2006).

\section{ConClusiones}

- El genotipo ST1 fue el más frecuente en los 36 aislados con $91.4 \%$, siendo la región de Lima la que concentra la mayor variabilidad de genotipos.

- Los resultados sugieren la presencia de tres nuevos perfiles alélicos STN.T 1, ST N.T 2 y ST N.T 3 en referencia con la base de datos de MLST.

- $\quad$ Se identificaron 19 alelos únicos y 229 sitios polimórficos en los cuatro genotipos descritos.

- El índice de asociación (IA) calculado fue de 4.7327 para las 36 cepas analizadas. 
Agradecimientos

El trabajo fue financiado y ejecutado en BIOSERVICE SRL. Los autores agradecen a MV Stephane Lovón Serrano y Rosario Condori por el apoyo logístico, sugerencias y revisión del manuscrito.

\section{Literatura Citada}

1. Amonsin A, Wellehan JF, Li LL, Vandamme P, Lindeman C, Edman M, Robinson RA, Kapur V. 1997. Molecular epidemiology of Ornithobacterium rhinotracheale. J Clin Microbiol 35: 2894-2898. doi: 10.1128 / JCM.35.11.2894-2898.1997

2. Chou CH, Lin SY, Chen CL, Tsai HJ. 2009. Use of random amplified polymorphic DNA analysis and singleenzyme amplified fragment length polymorphism in molecular typing of Ornithobacterium rhinotracheale strains. Avian Dis 53: 108-114. doi: 10.1637/8474-092708-Reg.1

3. Coll P, Coque MT, Domínguez MA, Vázquez J, Vila J. 2005. Procedimientos en microbiología clínica. Métodos moleculares de tipificación epidemiológica en Bacteriología. España: Sociedad Española de Enfermedades Infecciosas y Microbiología Clínica. 68 p.

4. Espinosa I, Colas M, Vichi J, Báez M, Martínez S. 2011. Isolation and identification of Ornithobacteriun rhinotracheale from laying hens in farms of la Habana province. Rev Salud Anim 33: 38-43.

5. Hafez MH, Reinhard S. 1999. Investigations on different Ornithobacterium rhinotracheale «ORT» isolates. Avian Dis 43.1: 1-7. doi:10.2307/1592755

6. Hafez HM, Vandamme P. 2011. Genus XXVI. Ornithobacterium. In: Bergey's manual of systematic bacteriology. $2^{\text {nd }}$ ed. New York: Springer. p 250-314.
7. Hassanzadeh M, Karrimi V, Fallah $N$, Ashrafi I. 2010. Molecular characterization of Ornithobacterium rhinotracheale isolated from broiler chicken flocks in Iran. Turk J Vet Anim Sci 34: 373-378. doi: 10.3906/vet-0810-19

8. Hung AL, Alvarado A. 2001. Phenotypic and molecular characterization of isolates of Ornithobacterium Rhinotracheale from Peru. Avian Dis 45. 4: 999-1005. doi: 10.2307/1592880

9. Jolley KA, Maiden MC. 2010. BIGSdb: scalable analysis of bacterial genome variation at the population level. BMC Bioinformatics 11: 595. doi: 10.1186/1471-2105-11-595

10. Jolley KA, Feil EJ, Chan MS, Maiden $M C$. 2001. Sequence type analysis and recombinational tests (START). Bioinformatics 17: 1230-1231. doi: 10.1093/bioinformatics/17.12.1230

11. Koga Y, Zavaleta A. 2005. Intraspecies genetic variability of Ornithobacterium rhinotracheale in commercial birds in Peru. Avian Dis 49: 108-111. doi: 10.1637/ 7235-070804R

12. Lillo A, Ashley FP, Palmer RM, Munson MA, Kyriacou L, Weightman AJ, Wade WG 2006. Novel subgingival bacterial phylotypes detected using multiple universal polymerase chain reaction primer sets. Oral Microbiol Immun 21: 61-68. doi: 10.1111/j.1399302X.2005.00255.x

13. Macagnan M. 2006. Caracterización fenotípica y genotípica de aislados de Ornithobacterium rhinotracheale de Brasil. Tesis de Maestría. Porto Alegre, Brasil: Univ. Federal de Rio Grande. 50 p.

14. Montes de Oca JR, Vega SV, Morales EV, Salgado MC, Blackhall JP, Soriano VE. 2018. Phylogenetic relationship of Ornithobacterium rhinotracheale strains. J Vet Med Sci 80: 869-873. doi: 10.1292/jvms. $17-0474$

15. Pubmlst.org. 2018. Ornithobacterium rhinotracheale. [Internet]. Available in: https://pubmlst.org/orhinotracheale/ 
16. Sakai E, Tokuyama Y, Nonaka F, Ohishi S, Ishikawa Y, Tanaka M, Taneno A. 2000. Ornithobacterium rhinotracheale infection in Japan: preliminary investigations. Vet Rec 146: 502-504.

17. Smith JM, Smith NH, O'Rourke M, Spratt BG. 1993. How clonal are bacteria? P Natl Acad Sci USA 90: 43844388. doi: 10.1073/pnas.90.10.4384

18. Soriano VE, Vera NA, Salado CR, Fernandez RP, Blackhall PJ. 2003. In vitro susceptibility of Ornithobacterium rhinotracheale to several antimicrobial drugs. Avian Dis 47: 476480. doi: 10.1637/0005-2086(2003)047[0476:IVSOOR]2.0.CO;2

19. Subaaharan S, Blackall LL, Blackall PJ. 2010. Development of a multi-locus sequence typing scheme for avian isolates of Pasteurella multocida. Vet Microbiol 141: 354-361. doi: 10.1016/ j.vetmic.2010.01.017

20. Tabatabai LB, Zimmerli MK, Zehr ES, Briggs RE, Tatum FM. 2010. Ornithobacterium rhinotracheale North American field isolates express a hemolysin-like protein. Avian Dis 54: 9941001. doi: 10.1637/9070-091409-Reg.1

21. Tamura K, Stecher G, Peterson D, Filipski A, Kumar S. 2013. MEGA6: molecular evolutionary genetics analysis version 6.0. Mol Biol Evol 30: 2725-2729. doi: 10.1093/molbev/mst197

22. Thieme S, Mühldorfer K, Gad W, Lüschow D, Hafez HM. 2016 . Molecular characterization of the recently emerged poultry pathogen Ornithobacterium rhinotracheale by multilocus sequence typing. PLoS One 11(9): e0163401. doi: 10.1371/journal.pone. 0163401

23. Thieme S, Hafez HM, Gutzera S, Warkentina $N$, Lüschowa $D$, Mühldorfer K. 2016b. Multilocus sequence typing of Ornithobacterium rhinotracheale isolated from pigeons and birds of prey revealed new insights into its population structure. Vet Anim Sci 1: 15-20. doi: 10.1016/j.vas.2016.10.002

24. Turan N, Ak S. 2002. Investigation of the presence of Ornithobacterium rhinotracheale in chickens in Turkey and determination of the seroprevalance of the infection using the enzyme-linked immunosorbent assay. Avian Dis 46: 442446. doi: 10.1637/0005-2086(2002)046[0442:IOTPOO]2.0.CO;2

25. Vandamme P, Segers P, Vancanneyt M, Van hove K, Mutters R, Hommez J,_Dewhirst F, et al. 1994. Description of Ornithobacterium rhinotracheale gen. nov. sp. nov. isolated from the avian respiratory tract. Int $\mathrm{J}$ Syst Evol Microbiol 44: 24-37 doi: 10.1099/ 00207713-44-1-24

26. Van Empel P, Van den Bosch H, Loeffen P, Storm P. 1997. Identification and serotyping of Ornithobacterium rhinotracheale. J Clin Microbiol 35: 418-421.

27. Van Empel P, Savelkoul P, Segers R; Stoof J, Loeffen P, Van den Bosch $H$. 1998. Molecular characterization of Ornithobacterium rhinotracheale. Utrecht: Universitei Utrecht. 3B:37- 48.

28. Van Empel PC, Hafez HM. 1999. Ornithobacterium rhinotracheale: a review. Avian Pathol 28: 217-227. doi: 10.1080/03079459994704

29. Soriano VE, Longinos GM, Fernández $\boldsymbol{R P}$. 2003. Isolation and characterization of Ornithobacteriun rhinotracheale from respiratory diseased turkeys. Vet México 34: 283-288.

30. Van Veen L, Hartman E, Fabri T. 2001. In vitro antibiotic sensitivity of strains of Ornithobacterium rhinotracheale isolated in The Netherlands between 1996 and 1999. Vet Rec 149: 611-613. doi: 10.1136/vr.149.20.611 